\title{
Influência do índice glicêmico dos alimentos sobre a concentração de marcadores pró-inflamatórios
}

\author{
Influence of the glycemic food index on the concentration of pros inflammatory markers \\ Influencia del índice glicémico de los alimentos sobre la concentración de marcadores pró-inflamatorios \\ Dallyla Jennifer Morais de SOUSA ${ }^{\mathbf{1}}$ \\ Larissa Layana Cardoso de SOUSA ${ }^{1}$ \\ Joanne Ribeiro RODRIGUES ${ }^{1}$ \\ Layla Rafaele Sampaio LEARTE ${ }^{2}$ \\ Gleyson Moura dos SANTOS ${ }^{3}$ \\ ${ }^{I}$ Curso de Graduação, Departamento de Nutrição, Centro de Ciências da Saúde, Universidade Federal do Piauí (UFPI). 64076-335. Teresina - PI, Brasil \\ ${ }^{2}$ Pós-Graduanda em Nutrição Clínica Funcional pelo Instituto de Pesquisas Ensino e Gestão em Saúde - IPGS \\ ${ }^{3}$ Nutricionista. Mestrando em Ciências e Saúde (PPGCS/UFPI). Pós-graduando em Fitoterapia Aplicada à Nutrição (UCAM). 64085-300. Teresina - PI, Brasil.
}

\section{Resumo}

Introdução: Dietas com alto índice glicêmico (IG) induzem hiperglicemia o que induz ao estresse oxidativo e aumenta as citocinas próinflamatórias, incluindo IL-6 e TNF- $\alpha$ em indivíduos saudáveis e com tolerância a glicose diminuída. Objetivo: Realizar levantamento de dados na literatura sobre a influência do IG dos alimentos nas concentrações de marcadores inflamatórios. Material e método: Para a seleção dos estudos foi realizada uma busca de publicações indexadas nas bases de dados Pubmed, Scielo, Cochrane e Lilacs utilizando-se a combinação dos termos cadastrados no site DeCS (Descritores em Ciências da Saúde): Glycemic Index and Inflammation. Os artigos selecionados foram publicados no período compreendido entre 2008 e 2017, obtendo-se um total de 781 artigos, dos quais foram selecionados 38, após a remoção de duplicatas a amostra se constituiu por 10 artigos que foram lidos na integra. Resultados: Dentre os estudos obtidos, observou-se que 6 retratam a relação de dietas com alto IG e o processo inflamatório, 2 relacionam o baixo IG com diminuição da inflamação e 2 por sua vez verificam tanto a relação da dieta de baixo IG como alto IG com o processo inflamatório. Conclusão: Foi demonstrado que uma dieta com alto IG aumenta as concentrações de marcadores inflamatórios como PCR, NF-kB e IL-6 e a dieta de baixo IG tem papel benéfico na inflamação crônica de baixo grau.

Descritores: Inflamação; Índice Glicêmico; Dieta de Alto Índice Glicêmico.

\section{Abstract}

Introduction: Diets with high glycemic index (GI) induce hyperglycemia which induces oxidative stress and increases proinflammatory cytokines, including IL-6 and TNF- $\alpha$ in healthy subjects with impaired glucose tolerance. Objective: To carry out data collection in the literature on the influence of the GI of the foods in the concentrations of inflammatory markers. Material and Method: For the selection of the studies a search of publications indexed in Pubmed, Scielo, Cochrane and Lilacs databases was done using the combination of the terms registered in the DeCS (Health Sciences Descriptors) site: Glycemic Index and Inflammation. The selected articles were published in the period between 2008 and 2017, obtaining a total of 781 articles, of which 38 were selected, after the removal of duplicates the sample consisted of 10 articles that were read in the whole. Results: Among the studies, it was observed that 6 portray the relationship of diets with high GI and the inflammatory process, 2 relate low GI with decreased inflammation and 2, in turn, verify both the relation of the low GI diet and the high GI diet IG with the inflammatory process. Conclusion: A high-GI diet has been shown to increase the concentrations of inflammatory markers such as CRP, NF-kB, and IL-6, and the low-GI diet plays a beneficial role in chronic low-grade inflammation.

Descriptors: Inflammation; Glycemic Index; Diet High Glycemic Index.

\section{Resumen}

Introducción: Dietas con alto índice glucémico (IG) induce hiperglicemia lo que induce al estrés oxidativo y aumenta las citocinas proinflamatorias, incluyendo IL-6 y TNF- $\alpha$ en sujetos sanos y con tolerancia a la glucosa disminuida. Objetivo: Realizar el levantamiento de datos en la literatura sobre la influencia del IG de los alimentos en las concentraciones de marcadores inflamatorios. Material y método: Para la selección de los estudios se realizó una búsqueda de publicaciones indexadas en las bases de datos Pubmed, Scielo, Cochrane y Lilacs utilizando la combinación de los términos registrados en el sitio DeCS (Descriptores en Ciencias de la Salud): Glycemic Index and Inflammation. Los artículos seleccionados fueron publicados en el período comprendido entre 2008 y 2017, obteniéndose un total de 781 artículos, de los cuales fueron seleccionados 38, después de la remoción de duplicados la muestra se constituyó por 10 artículos que fueron leídos en la integración. Resultados: Entre los estudios obtenidos, se observó que 6 retrata la relación de dietas con alto IG y el proceso inflamatorio, 2 relacionan el bajo IG con disminución de la inflamación y 2 a su vez verifican la relación de la dieta de bajo IG como alto IG con el proceso inflamatorio. Conclusión: Se ha demostrado que una dieta con alto IG aumenta las concentraciones de marcadores inflamatorios como PCR, NF-kB e IL-6 y la dieta de bajo IG tiene un papel benéfico en la inflamación crónica de bajo grado.

Descriptores: Inflamación; Índice Glucémico; Dieta de Alto Índice Glucémico.

INTRODUÇÃO

O sistema imunológico possui proteínas que são responsáveis por mediar suas respostas, tornando-as mais eficazes. As citocinas são produzidas principalmente por macrófagos e monócitos, elas agem como mediadores das respostas inflamatória e imune e atuam no crescimento e diferenciação de outras células, podendo exercer mecanismo reguladores positivos ou negativos sobre essas respostas ${ }^{1}$.

A inflamação crônica geralmente começa de maneira insidiosa, como resposta de baixo grau, latente e assintomática provocando lesão tissular incipiente por meio da ativação, em longo prazo, do sistema imune inato, podendo causar posterior manifestação de doenças crônicas não transmissíveis, como as cardiovasculares, o diabetes mellitus, a obesidade, o câncer, entre outras ${ }^{2}$. O aumento da inflamação provoca aumento na síntese de células, citocinas e proteínas próinflamatórias, como neutrófilos, monócitos, IL-6, TNFa e PCR, dentre outras. $\mathrm{O}$ aumento de neutrófilos e macrófagos em resposta à inflamação provoca uma maior síntese de IL-6 que, por sua vez, aumenta a produção de PCR no fígado ${ }^{3}$.

O índice glicêmico (IG) é uma medida do impacto relativo do carboidrato presente nos alimentos e na concentração de glicose plasmática, determinando o seu efeito sobre a glicemia pós-prandial ${ }^{4}$. Tal índice indica, indiretamente, como o alimento se comporta em termos de velocidade de digestão e absorção. Assim, alimentos com maior IG promovem maior aumento na resposta glicêmica e insulínica, enquanto os de baixo IG estão associados com menores respostas ${ }^{2}$.

Estudos relatam que dietas com alto IG 
induzem hiperglicemia o que induz ao estresse oxidativo e aumenta as citocinas pró-inflamatórias, incluindo IL-6 e TNF- $\alpha$ em indivíduos saudáveis e com tolerância a glicose diminuída. O aumento do nível de citocinas pró-inflamatórias causa rupturas na sinalização da insulina, levando subsequentemente à resistência à insulina ${ }^{5}$.

Diante disso, o objetivo deste estudo foi realizar um levantamento de dados sobre a influência do índice glicêmico dos alimentos nas concentrações de marcadores inflamatórios.

\section{MATERIAL E MÉTODO}

O estudo caracteriza-se como uma revisão da literatura apresentando informações relevantes a cerca da influência do índice glicêmico dos alimentos sobre marcadores inflamatórios. Para a seleção dos estudos foi realizada uma busca de publicações indexadas nas bases de dados Pubmed, Scielo, Cochrane e Lilacs utilizandose a combinação dos termos cadastrados no site DeCS (Descritores em Ciências da Saúde): Glycemic Index and Inflammation. Os artigos selecionados foram publicados no período compreendido entre 2008 e 2017. Foram utilizados como critérios de inclusão: estudos que avaliassem o índice glicêmico dos alimentos sobre marcadores inflamatórios como NF-K $\beta$, TNF- $\alpha$, IL-6 e PCR, estudos originais, realizados com humanos e publicados em período de 2008 a 2018 nos idiomas inglês, português e espanhol. Foram excluídos estudos de revisão, realizados em animais e que estavam fora do recorte temporal ou da temática pré-estabelecida (Figura 1). Para a extração de dados dos artigos selecionados procedeu-se a esquematização em tabela na qual estavam especificados os seguintes itens: autores e ano da publicação, intervenção utilizada e resultados obtidos. A análise dos dados foi realizada de forma descritiva.

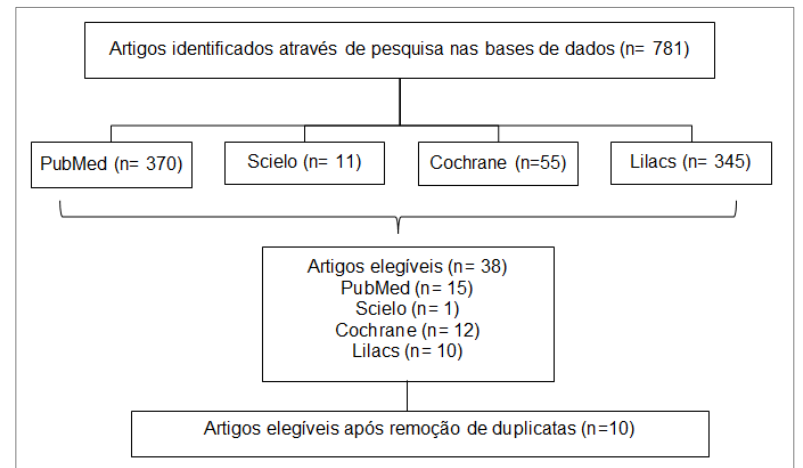

Figura 1. Fluxograma de identificação e seleção dos artigos para revisão sobre a influência do índice glicêmico dos alimentos na concentração de marcadores pró-inflamatórios (Fonte: Dados da Pesquisa).

\section{RESULTADOS}

O Quadro 1 apresenta uma visão geral dos estudos levantados na literatura que retratam a influência do IG dos alimentos em marcadores próinflamatórios e consequentemente no processo de inflamação crônica de baixo grau.

\section{DISCUSSÃO}

Dentre os estudos selecionados 6 relacionaram o alto IG com a inflamação, 2 demonstram diminuição do processo inflamatório a partir da adesão a dietas de baixo IG e 2 fizeram as duas relações concomitantemente. No estudo de Dickinson et al. ${ }^{6}$ realizado com 17 pessoas, sendo 9 mulheres e 8 homens, foi observado que uma fonte de carboidrato com alto IG tem a mesma capacidade de ativar agudamente o NF-kB que um teste de glicose oral de 50 g. E por outro lado, uma fonte de carboidrato de baixo IG teve um efeito quase desprezível na ativação de NF-B.

Quadro 1. Estudos que investigaram a influência do IG no processo inflamatório crônico.

\begin{tabular}{|c|c|c|}
\hline Autor & Descricão do estudo & Resultados \\
\hline $\begin{array}{c}\text { Dickinson } \\
\text { et al.6 } \\
2008\end{array}$ & $\begin{array}{l}\text { Examinaram as diferenças agudas } \\
\text { na ativação pós-prandial de NF- } \\
\mathrm{kB} \text { em células mononucleares de } \\
\text { indivíduos saudáveis após cargas } \\
\text { fisiológicas de carboidratos } \\
\text { diferindo no IG. }\end{array}$ & $\begin{array}{c}\text { Constataram que uma fonte de } \\
\text { carboidrato com alto IG tem a mesma } \\
\text { capacidade de ativar agudamente o NF- } \\
\text { kB que um teste de glicose oral de } 50 \text { g. } \\
\text { Por outro lado, uma fonte de } \\
\text { carboidrato de baixo IG teve um efeito } \\
\text { quase desprezível na ativação de NF-B. }\end{array}$ \\
\hline $\begin{array}{c}\text { Du et al. }{ }^{7} \\
2008\end{array}$ & $\begin{array}{c}\text { Investigaram a relação entre IG e } \\
\text { carga glicêmica com a ingestão de } \\
\text { alimentos e nutrientes } \\
\text { explorando sua associação com } \\
\text { fatores de risco metabólicos, } \\
\text { incluindo marcadores de controle } \\
\text { glicêmico, sensibilidade à } \\
\text { insulina, metabolismo lipídico e } \\
\text { inflamação crônica sistêmica. }\end{array}$ & $\begin{array}{l}\text { Observaram que um aumento de } 10 \\
\text { unidades no IG foi associado a um } \\
\text { decréscimo de } 7 \% \text { na concentração de } \\
\text { colesterol HDL, um aumento de 10\% na } \\
\text { relação entre colesterol total e } \\
\text { colesterol HDL e um aumento de } 29 \% \\
\text { na concentração de PCR. }\end{array}$ \\
\hline $\begin{array}{c}\text { Levitan } \\
\text { et al. }^{8} \\
2008\end{array}$ & $\begin{array}{c}\text { Examinaram a associação } \\
\text { transversal de IG e carga } \\
\text { glicêmica dietéticos com lipídios } \\
\text { sanguíneos e PCR em mulheres } \\
\text { não diabéticas de meia-idade e } \\
\text { idosas, avaliando se essas } \\
\text { relações variavam de acordo com } \\
\text { o índice de massa corporal (IMC). }\end{array}$ & $\begin{array}{l}\text { Constataram que o IG da dieta foi } \\
\text { associado a pequenos aumentos no } \\
\text { colesterol LDL, razão do colesterol } \\
\text { LDL:HDL, TG e PCR e com uma } \\
\text { pequena diminuição no colesterol HDL. }\end{array}$ \\
\hline $\begin{array}{c}\text { Buyken et } \\
\text { al.9 } \\
2010\end{array}$ & $\begin{array}{c}\text { Examinaram se o IG alimentar e } \\
\text { o consumo de grupos alimentares } \\
\text { contendo fibras e carboidratos } \\
\text { estavam associados à mortalidade } \\
\text { por doença inflamatória de } 13 \\
\text { anos em uma coorte australiana } \\
\text { mais velha. }\end{array}$ & $\begin{array}{l}\text { Observaram que os participantes que } \\
\text { morreram de doenças relacionadas à } \\
\text { inflamaçẫo apresentavam maior IG } \\
\text { alimentar. }\end{array}$ \\
\hline $\begin{array}{c}\text { Gogebakan } \\
\text { et al. }^{10} \\
2011\end{array}$ & $\begin{array}{l}\text { Investigaram se as melhorias na } \\
\text { proténa C reativa de alta } \\
\text { sensibilidade (PCR-US), } \\
\text { triglicérides, colesterol total, HDL } \\
\text { e LDL assim como a pressão } \\
\text { arterial após o período inicial de } \\
\text { perda de peso poderiam ser } \\
\text { mantidos ou melhorados com } \\
\text { dietas que diferiam no conteúdo } \\
\text { proteico e glicêmico. }\end{array}$ & $\begin{array}{l}\text { Observaram que durante a intervenção } \\
\text { dietética, a diminuição na PCR-US dos } \\
\text { participantes nos grupos de baixo IG foi } \\
\text { maior em comparação com os grupos } \\
\text { de alto IG. }\end{array}$ \\
\hline $\begin{array}{l}\text { Kelly } \\
\text { et al. } \\
2011\end{array}$ & $\begin{array}{c}\text { Avaliaram se uma intervenção } \\
\text { através de uma dieta com baixo } \\
\text { IG combinada com exercícios } \\
\text { aeróbicos reduziria a liberação de } \\
\text { glicose pós-prandial atenuando a } \\
\text { hiperglicemia prevalente que } \\
\text { induz inflamação de baixo grau e } \\
\text { secreção de citocinas em células } \\
\text { mononucleares (MNC). }\end{array}$ & $\begin{array}{l}\text { Verificaram que as concentrações } \\
\text { plasmáticas de MNC e TNFa tenderam } \\
\text { a ser maiores no grupo em uso de dieta } \\
\text { com alto IG; e menores no grupo em } \\
\text { uso da dieta de baixo IG, além disso } \\
\text { nesse mesmo grupo foi observado uma } \\
\text { diminuição significativa na secreção de } \\
\text { IL-6. }\end{array}$ \\
\hline $\begin{array}{c}\text { Bullo } \\
\text { et al. }^{12} \\
2013\end{array}$ & $\begin{array}{c}\text { Examinaram como o índice } \\
\text { glicêmico dietético e a carga } \\
\text { glicêmica da dieta estão } \\
\text { associados a várias adipocinas e } \\
\text { correlatos marcadores de risco } \\
\text { metabólico de obesidade e } \\
\text { diabetes de maneira transversal e } \\
\text { longitudinal. }\end{array}$ & $\begin{array}{l}\text { Observaram que indivíduos nos quartis } \\
\text { mais altos do IG mostraram níveis } \\
\text { significativamente mais elevados de } \\
\text { TNF e IL-6 do que aqueles nos quartis } \\
\text { mais baixos. O IG dietético foi } \\
\text { negativamente relacionado aos níveis } \\
\text { plasmáticos de leptina e adiponectina. }\end{array}$ \\
\hline $\begin{array}{c}\text { Goletzke } \\
\text { et al. }{ }^{13} \\
2014\end{array}$ & $\begin{array}{c}\text { Analisaram a associação } \\
\text { prospectiva entre quantidade e } \\
\text { qualidade do consumo habitual } \\
\text { de carboidratos na dieta durante } \\
\text { a puberdade e marcadores pró- } \\
\text { inflamatórios, bem como a } \\
\text { adiponectina em uma coorte de } \\
\text { jovens alemães saudáveis. }\end{array}$ & $\begin{array}{l}\text { Verificaram que apenas a ingestão de } \\
\text { carboidratos de fontes de IG mais alto } \\
\text { foi relevante para concentrações } \\
\text { posteriores de IL-6. }\end{array}$ \\
\hline $\begin{array}{c}\text { Rouhani } \\
\text { et al. }^{14} \\
2016\end{array}$ & $\begin{array}{l}\text { Examinaram o efeito de uma } \\
\text { dieta de baixo IG sobre a } \\
\text { concentracãao de insulina, } \\
\text { glicemia de jejum, marcadores } \\
\text { inflamatórios e adiponectina } \\
\text { entre adolescentes do sexo } \\
\text { feminino saudáveis, acima do } \\
\text { peso e obesas. }\end{array}$ & $\begin{array}{c}\text { Obtiveram que a concentração sérica } \\
\text { média de PCR-US no grupo com dieta } \\
\text { de baixo IG diminuiu } \\
\text { significativamente após intervenção de } \\
10 \text { semanas. }\end{array}$ \\
\hline $\begin{array}{c}\text { Gomes } \\
\text { et al. }{ }^{15} \\
2017\end{array}$ & $\begin{array}{c}\text { Avaliaram o efeito do consumo de } \\
\text { dietas de alto ou baixo IG por } 30 \\
\text { dias consecutivos em } \\
\text { antropometria, composição } \\
\text { corporal, ingestão de alimentos, } \\
\text { controle glicêmico e lipídico, } \\
\text { marcador inflamatório em } \\
\text { concentrações em pacientes com } \\
\text { diabetes tipo } 2 .\end{array}$ & $\begin{array}{l}\text { Constataram que o consumo de uma } \\
\text { dieta de alto IG por } 30 \text { dias } \\
\text { consecutivos causou um aumento nas } \\
\text { concentrações de frutosamina, NEFA e } \\
\text { TNF- } \alpha \text {, enquanto que uma dieta com } \\
\text { baixo IG causou uma redução } \\
\text { significativa de aproximadamente } 2 \% \\
\text { na gordura corporal entre pacientes } \\
\text { com sobrepeso com tipo } 2 \text { diabetes. }\end{array}$ \\
\hline
\end{tabular}

O ensaio clínico paralelo, multicêntrico controlado, aleatorizado, de 6 anos de Bullo et al. ${ }^{12}$ realizado com 511 homens e mulheres idosos, 
demonstrou a influência do IG na expressão de marcadores inflamatórios. Os indivíduos nos quartis mais altos do IG mostraram níveis significativamente mais elevados de TNF e IL-6 do que aqueles nos quartis mais baixos. Resultados semelhantes foi observado no estudo de coorte de Goletzke et al. ${ }^{13}$ onde a partir da análise de registros alimentares e amostras de sangue de 205 pessoas foi verificado que uma maior ingestão de carboidratos de fontes alimentares de maior IG pode ser prejudicial e maior consumo de grãos integrais pode ser benéfico para concentrações adultas de IL-6.

Gomes et al. ${ }^{15}$ em seu ensaio paralelo controlado e randomizado realizado com 20 pessoas portadoras de diabetes tipo 2, verificou que o consumo de uma dieta de alto IG por 30 dias consecutivos provocou um aumento nas concentrações de frutosamina, NEFA e TNF- $\alpha$, enquanto que uma dieta com baixo IG ocasionou uma redução significativa de aproximadamente $2 \%$ na gordura corporal entre pacientes com sobrepeso. Tais resultados sugerem que o consumo de dietas de baixo IG pode ajudar a reduzir a gordura corporal e prevenir as alterações inflamatórias e metabólicas prejudiciais induzidas pelas dietas de alto índice glicêmico.

O estudo de coorte populacional com amostra constituída por 2735 participantes de Buyken et al. ${ }^{9}$ foi observado uma ligação potencialmente importante entre o IG alimentar e a morte atribuída à patologia de inflamação, estresse oxidativo e origens infecciosas.

Foi demonstrado o papel benéfico das dietas de baixo IG na otimização da sensibilidade à insulina, metabolismo lipídico, inflamação crônica e, em não fumantes, glicemia de jejum pelo estudo de coorte populacional de Du et al. ${ }^{7}$ que analisaram uma amostra composta por 517 homens e 457 mulheres em que $30 \%$ deles tinham diagnóstico recente de diabetes tipo 2. Da mesma forma, Gogebakan et al. ${ }^{10}$ através de um estudo de coorte com 932 adultos com sobrepeso verificaram que uma dieta com baixo índice glicêmico sustentada por uma dieta com baixo teor proteico pode reduzir ainda mais a PCR e, consequentemente, a inflamação de baixo grau. Os autores relacionaram esse resultado com a redução dos níveis de glicose pós-prandial que estimularia a expressão de genes inflamatórios por mecanismos epigenéticos.

De acordo com o estudo duplo-cego controlado por placebo e randomizado de Levitan et al. ${ }^{8}$ realizado com 18.137 mulheres saudáveis com idade $\geq$ 45 anos sem diagnóstico de diabetes, a quantidade e a qualidade dos carboidratos consumidos podem influenciar as concentrações de lipídios no sangue e a inflamação. Dietas caracterizadas por baixo IG e GL foram associadas com perfis lipídicos um pouco mais favoráveis e menor PCR. Os autores atribuíram esse resultado à estimulação da liberação de hormônios contrarreguladores que ocorre 4 a 6 horas após uma refeição com um alto IG, que aumentam as concentrações de glicose e ácidos graxos livres induzindo resistência à insulina que parece causar aumento dos TG e mediadores inflamatórios e diminuição do colesterol HDL.
O estudo randomizado de Kelly et al. ${ }^{11}$ com 28 idosos com resistência a insulina demonstrou que a adesão a uma dieta com baixo índice glicêmico em combinação com exercícios aeróbicos pode reverter os efeitos da obesidade sobre a produção de citocinas próinflamatórias. De modo similar, no ensaio clinico randomizado de Rouhani et al. ${ }^{14}$ com estudantes do sexo feminino entre 12 e 18 anos com sobrepeso ou obesidade foi constatado que a adesão a uma dieta com baixo índice glicêmico pode ter efeito favorável sobre a inflamação.

Todos os estudos relacionados nesta revisão independentemente do tipo de pesquisa, sujeitos participantes, número amostral e método de intervenção, apontam a relevância da influência do IG na inflamação crônica de baixo grau. Dietas com alto IG têm sido associadas a doenças, enquanto dietas de baixo IG são consideradas protetivas, devido provavelmente à redução das excursões de glicose pós-prandial.

Sabe-se que alimentos com alto IG induzem maior concentração de glicose, diante disso um dos mecanismos apontados pela literatura para explicar o efeito inflamatório desse tipo de dieta está relacionado com a capacidade das células mononucleadas de usar glicose para a produção de NADPH que quando oxidado, resulta na geração de espécies reativas de oxigênio, ativando a via do $\mathrm{NF}-\mathrm{kB}$ e resultando em aumento do $\mathrm{TNF}^{16-18}$. O TNF $\alpha$ circulante se liga aos seus receptores, induzindo a ativação de serina-quinases que podem estimular a transcrição de genes inflamatórios, levando ao aumento da produção de proteínas inflamatórias nos tecidos-alvo, incluindo músculo, tecido adiposo e fígado ${ }^{19,20}$.

Os estudos demonstram que dietas com alto IG podem aumentar os marcadores inflamatórios e da síndrome metabólica, como o NFkB pós-prandial em células mononucleares, proteína $\mathrm{C}$ reativa altamente sensível (PCR-US), interleucinas (IL) -6, IL -7, IL-18 e níveis de radicais livres; enquanto dietas de baixo IG promovem melhor sensibilidade à insulina, menor pressão sanguínea e colesterol total ${ }^{21}$.

\section{CONCLUSÃO}

Diante do que foi exposto pela literatura podese inferir que o IG apresenta grande relevância quando se trata de inflamação crônica de baixo grau, visto que foi demonstrado que uma dieta com alto IG aumenta as concentrações de marcadores inflamatórios como NF$\mathrm{k} \beta$, PCR e IL-6. Os estudos também apontam que a adoção de uma dieta com baixo IG possui efeitos benéficos contra o processo inflamatório promovendo melhor qualidade de vida.

\section{REFERÊNCIAS}

1. Mayer LF, Bona KS, Abdalla FH, Almeida FL, Pozzobon RCR, Charão MF et al. Perspectivas laboratoriais na avaliação da resposta inflamatória. Rev Bras Farm. 2010;91(4):149-61.

2. Lima RPE, Caetano CL, Nader DA Avaliação dos níveis séricos de proteína C-reativa em indivíduos com periodontite: um estudo piloto. Braz J Periodontol. 2016;6(1):13-9. 
3. Teixeira BC, Lopes AL, Macedo RCO, Correa CS, Ramis TR, Ribeiro JL et al. Marcadores inflamatórios, função endotelial e riscos cardiovasculares. J vasc bras. 2014;13(2):108-15.

4. Cunha LS. Análise dos efeitos de dietas baseadas no índice glicêmico em diabéticos tipo 2 [monografia]. Brasília: Faculdade de Ciências da Educação e Saúde; 2013.

5. Kim Y, Chen J, Wirth MD, Shivappa N, Herbert JR. Lower dietary inflammatory index scores are associated with lower glycemic index scores among college students. Nutrients. 2018; 10(2):182.

6. Dickinson S, Hancock DP, Petocz P, Ceriello A, Brand-Miller J. High-glycemic index carbohydrate increases nuclear factor-kappaB activation in mononuclear cells of young, lean healthy subjects. Am J Clin Nutr. 2008; 87(5):1188-93.

7. Du H, Van Der ADL, Van Bakel MIM, Van Der Kallen CJ, Blaak EE, Van Greevenbroek MM et al. Glycemic index and glycemic load in relation to food and nutrient intake and metabolic risk factors in a Dutch population. Am J Clin Nutr. 2008;87(3):655-61.

8. Levitan EB, Cook NR, Stampfer MJ, Ridker PM, Rexrode KM, Buring JE et al. Dietary glycemic index, dietary glycemic load, blood lipids, and $\mathrm{C}$ reactive protein. Metabolism. 2008;57(3):437-43.

9. Buyken AE, Flood V, Empson M, Rochtchina E, Barclay AW, Brand-Miller $\mathrm{J}$ et al. Carbohydrate nutrition and inflammatory disease mortality in older adults. Am J Clin Nutr. 2010;92(3):634-43.

10. Gogebakan O, Kohl A, Osterhoff MA, Van Baak MA, Jebb SA, Papadaki A et al. Effects of weight loss and long-term weight maintenance with diets varying in protein and glycemic index on cardiovascular risk factors. Circulation. 2011; 124(25):2829-38.

11. Kelly KR, Haus JM, Solomon TP, Patrick-Melin AJ, Cook M, Rocco M et al. A low-glycemic index diet and exercise intervention reduces $\mathrm{TNFa}$ in isolated mononuclear cells of older, obese adults. J Nutr. 2011;141(6):1089-94.

12. Bullo M, Casas R, Portillo MP, Basora J, Estruch R, Garcia-Arellano A et al. Dietary glycemic index/load and peripheral adipokines and inflammatory markers in elderly subjects at high cardiovascular risk. Nutr Metab Cardiovasc Dis. 2013;23(5):443-50.

13. Goletzke J, Buyken AE, Joslowski G, Bolzenius K, Remer $\mathrm{T}$, Carstensen $\mathrm{M}$ et al. Increased intake of carbohydrates from sources with a higher glycemic index and lower consumption of whole grains during puberty are prospectively associated with higher IL6 concentrations in younger adulthood among healthy individuals. J Nutr. 2014;144(10):1586-93.

14. Rouhani MH, Kelishadi R, Hashemipour M, Esmailzadeh A, Surkan PJ, Keshavarz A et al. The impact of a low glycemic index diet on inflammatory markers and serum adiponectin concentration in adolescent overweight and obese girls: a randomized clinical trial. Horm Metab Res. 2016;48(4):251-56.

15. Gomes JMG, Fabrini SP, Alfenas RCG. Low glycemic index diet reduces body fat and attenuates inflammatory and metabolic responses in patients with type 2 diabetes. Arch Endocrinol Metab. 2017;61(2):137-44.

16. Mohanty P, Hamouda W, Garg R, Aljada A, Ghanim H, Dandona P. Glucose challenge stimulates reactive oxygen species (ROS) generation by leucocytes. J Clin Endocrinol Metab. 2000;85(8):2970-73.

17. Evans JL, Goldfine ID, Maddux BA, Grodsky GM. Oxidative stress and stress-activated signaling pathways: a unifying hypothesis of type 2 diabetes. Endocr Rev. 2002;23(5):599-622.

18. Ghanim H, Aljada A, Hofmeyer D, Syed T, Mohanty P, Dandona P. Circulating mononuclear cells in the obese are in a proinflammatory state. Circulation. 2004;110(12):1564-71.

19. Hotamisligil GS. Inflammatory pathways and insulin action. Int J Obes Relat Metab Disord. 2003;27 Suppl 3:S53-5.

20. Perseghin G, Petersen K, Shulman GI. Cellular mechanism of insulin resistance: potential links with inflammation. Int $\mathbf{J}$ Obes Relat Metab Disord. 2003;27 Suppl 3:S6-11.

21. Bosma-den Boer MM, van Wetten ML, Pruimboom L. Chronic inflammatory diseases are stimulated by current lifestyle: how diet, stress levels and medication prevent our body from recovering. Nutr Metab (Lond). 2012;9(1):32.

\section{CONFLITO DE INTERESSES}

Os autores declaram não haver conflitos de interesse.

\section{AUTOR PARA CORRESPONDÊNCIA}

\section{Gleyson Moura dos Santos}

g_leyson_moura@hotmail.com 